\title{
LUMINESCENCE AND TRANSIENT ABSORPTION OF $\mathrm{CdWO}_{4}$ CRYSTALS*
}

\author{
D. Millers, L. Grigorieva and S. Chernov \\ Institute of Solid State Physics, University of Latvia \\ 8 Kengaraga str., 1063, Riga, Latvia. \\ (Received July 23, 1998; revised version February 4, 1999)
}

\begin{abstract}
Luminescence and transient absorption of the oxygen-deficient $\mathrm{CdWO}_{4}$ crystal were studied. The electron transitions from the excited state of the luminescence center to some higher energy state are responsible for the transient absorption observed. The scheme of luminescence center levels is proposed from the obtained experimental results.
\end{abstract}

PACS numbers: 78.60. Ya, 78.40.--q

\section{Introduction}

$\mathrm{CdWO}_{4}$ (CWO) crystals are used as scintillators for X-ray detection in the computed tomography [1]. However the mechanism of scintillation in CWO is not clear up to now, moreover different undoped CWO crystals studied by several authors showed considerably different decay times of luminescence [2]. Thus the defects incorporated in crystals during growth change the luminescent properties considerably. Since the crystals were undoped the composition of crystal can be responsible for these defects. Recent study [2] of oxygen deficient CWO crystals shows an additional luminescence band centered at $\approx 2.0 \mathrm{eV}$ and it was concluded that the oxygen-deficient defects were responsible for this luminescence. In. the present investigation, the time-resolved study of luminescence and transient absorption was carried out for CWO oxygen-deficient crystals.

\section{Experimental}

Two different equipment sets were used for the luminescence studies. One excitation source was a pulsed nitrogen laser and the registration was carried out by time-correlated photon counting. Another excitation source was a pulsed electron beam and PMT output signal was displayed by a storage oscilloscope. Both excitation sources had a pulse width 10 ns. The luminescence spectra and decay kinetics were measured under both kinds of excitation.

*The results of this paper were initially presented at The Jablonski Centennial Conference on Luminescence and Photophysics, July 23-27, 1998, Toruń, Poland. 
The transient absorption was studied under electron beam irradiation. The experimental setup was described in detail elsewhere [2]. All measurements were completed at $300 \mathrm{~K}$.

The studied CWO crystals were grown in the Institute of Solid State Physics, University of Latvia. Two types of crystals were used: uncolored, close to the stoichiometric, and yellow hue, oxygen deficient.

\section{Results and discussion}

The luminescence spectrum of uncolored CWO crystals under laser beam excitation is the same as under electron beam excitation at $300 \mathrm{~K}$ (Fig. 1, curve 1). This spectrum reveals one wide band peaking at $2.5 \mathrm{eV}$. The same band was observed by Deych et al. [1]. The oxygen deficient CWO crystals have a yellow hue. The luminescence spectrum of oxygen deficient CWO crystals is shown in Fig. 1 (curves 2 and 3). This spectrum reveals two overlapping bands: one at $\approx 2.5 \mathrm{eV}$ and another at $\approx 2.0 \mathrm{eV}$. The band at $2.0 \mathrm{eV}$ is due to some oxygen deficiency defect [2] and this band is dominant under laser beam excitation, whereas under electron beam excitation the $2.5 \mathrm{eV}$ band was dominant and a small contribution of $2.0 \mathrm{eV}$ band was observed (Fig. 1). Spectra are different because under laser beam excitation ( $3.67 \mathrm{eV}$ ) the luminescence centers due to oxygen deficit were excited whereas the regular intrinsic centers were excited as well by electron beam.

The kinetics of luminescence decay within the $2.5 \mathrm{eV}$ band coincides in both - uncolored and yellow hue crystals. These kinetics were the same as observed in [2]: under a laser beam excitation first-order (exponential) decay takes place $(\tau=15 \mu \mathrm{s}$ ), whereas under electron beam irradiation the decay kinetics can be well approximated by two exponential components with time constants of $2 \mu \mathrm{s}$ and

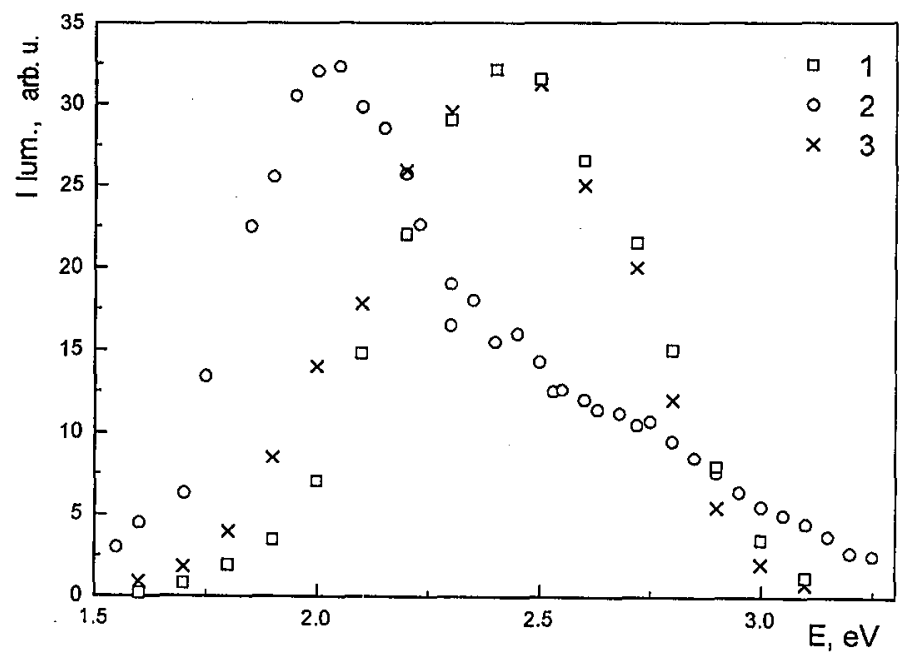

Fig. 1. Luminescence spectra of CWO colorless (1) and yellow hue crystals $(2,3) .1,2$ - under laser excitation and 3 - under electron beam excitation. 
$11 \mu \mathrm{s}$. Similar two-component luminescence decay was observed in CWO crystals under $\gamma$-ray excitation at $300 \mathrm{~K}[3]$.

The luminescence decay study of yellow hue crystal within $2.0 \mathrm{eV}$ band shows a similar result. The kinetics of luminescence decay within the $2.0 \mathrm{eV}$ band under laser beam excitation shows exponential decay with time constant $\tau=$ $26 \mu \mathrm{s}$, whereas under electron beam irradiation two exponents with time constants $\tau_{1}=0.6 \mu$ s and $\tau_{2}=11.5 \mu$ s were observed (Fig. 2a). One can point out that decay with a time constant $\tau=11.5 \mu$ s may be due to a large contribution of the $2.5 \mathrm{eV}$ band over the whole $2.0 \mathrm{eV}$ band and the used equipment (oscilloscope)
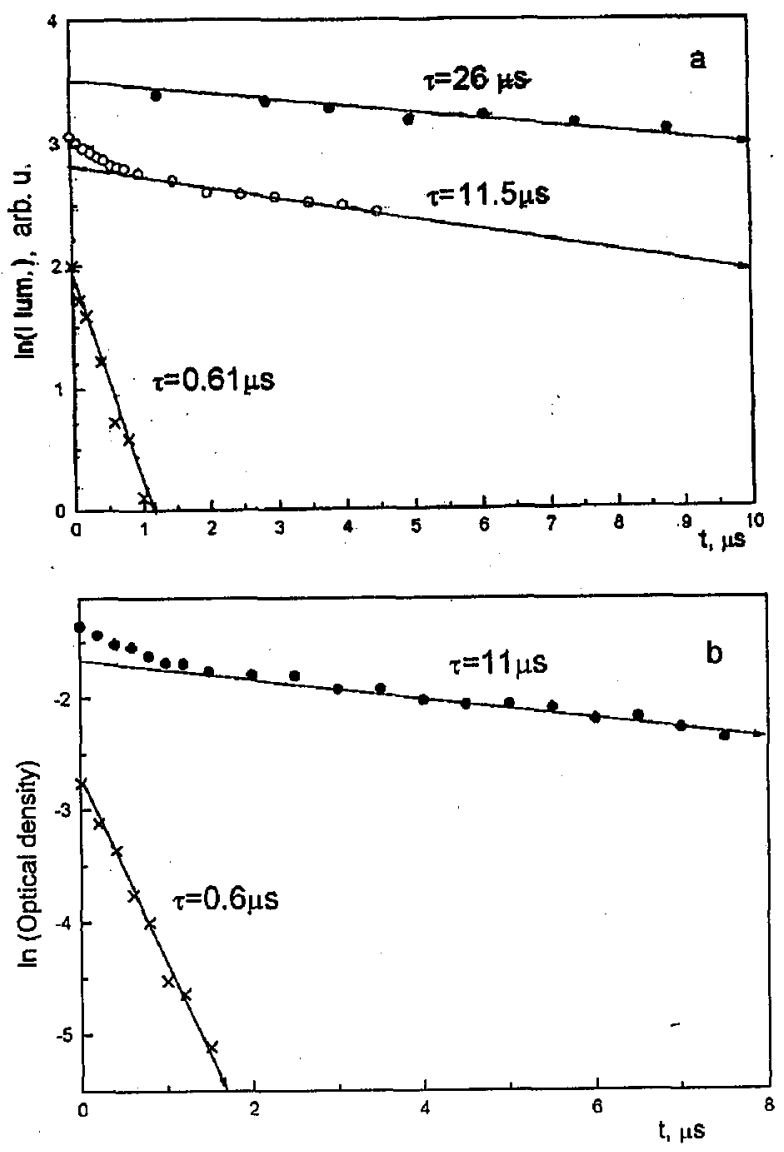

Fig. 2. Decay kinetics of luminescence at $1.8 \mathrm{eV}$ (a) and transient absorption at $1.8 \mathrm{eV}$ (b) at $300 \mathrm{~K}$. Solid lines are the theoretical approximation with exponents. (a) Filled circles is experiment under laser excitation, open circles is experiment under electron beam excitation; crosses - the fast decay component of luminescence under electron beam excitation. (b) Filled circles is experimental transient absorption decay kinetics; crosses is transient absorption fast decay component. 
did not allow to resolve a weak contribution of the slower decay ( $\tau=26 \mu \mathrm{s}$ ) of the $2.0 \mathrm{eV}$ band. However the decay kinetics of luminescence within the $2.5 \mathrm{eV}$ band shows similar result [2] — the luminescence excited by laser beam decays slower than that excited by electron beam. Figure 2 shows the kinetics measured at most advantageous photon energy of $1.8 \mathrm{eV}$, as it is seen from Fig. 1. The contribution of fast component in luminescence decay is about $21 \%$ of the total intensity at $1.8 \mathrm{eV}$. The decay times of the luminescence indicates that electron transitions responsible for emission are partially forbidden. Two components in luminescence decay kinetics observed under ionizing irradiation gives evidence that some number of luminescence centers are perturbed by unknown radiation defects (fast component of the luminescence decay) whereas other luminescence centers are not perturbed by radiation defects (slow component of the luminescence decay).

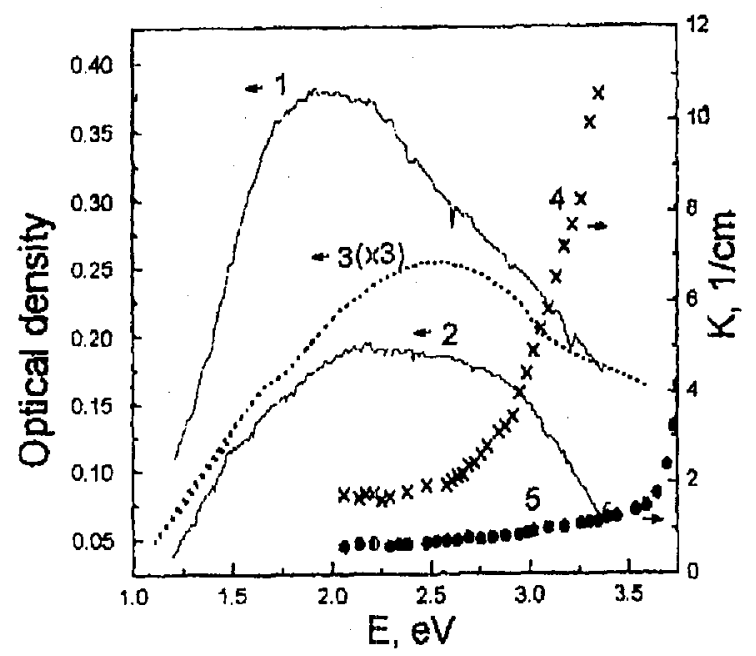

Fig. 3. Transient absorption spectra for yellow hue crystal (1 - at the end of irradiation pulse and 2 - at $5.5 \mu$ s delay) and uncolored crystal (3). The fundamental absorption edge for yellow hue crystal (4) and uncolored crystal (5).

The transient absorption spectra induced by electron beam irradiation are shown in Fig. 3 (curves 1 and 2 for yellow hue sample and curve 3 for uncolored sample). The spectra in yellow hue sample reveal at least two overlapping bands at $\approx 2.0 \mathrm{~V}$ and at $\approx 2.5 \mathrm{eV}$. The band at $2.0 \mathrm{eV}$ is dominant at the end of irradiation pulse, however amplitudes of the $2.0 \mathrm{eV}$ and $2.5 \mathrm{eV}$ bands are nearly the same at $5.5 \mu \mathrm{s}$ delay (Fig. 3, curve 2). The relaxation of the absorption at $1.8 \mathrm{eV}$ shows two exponential components with time constants $0.6 \mu$ s and $11 \mu \mathrm{s}$ (Fig. 2b). The contribution of the fast component $(0.6 \mu \mathrm{s})$ is about $24 \%$ of the total optical density observed at the end of the irradiation pulse. Comparison of the contribution of the fast components in absorption and luminescence decay shows that these are slightly different (Fig. 2a, b).

The coincidence of the time constants of the transient absorption and luminescence decay kinetics for yellow hue crystal leads to the conclusion that both - 
the absorption at $2.0 \mathrm{eV}$ and luminescence at $2.0 \mathrm{eV}$ - in oxygen-deficient CWO crystals arise from the same center state, and the observed transient absorption is an excited state absorption of the luminescence centers. Since the oxygen-deficient centers are responsible for this luminescence, the excited state absorption is due to the same centers. Similar excited state absorption is observed in CWO for intrinsic luminescence centers [2], as well as in $\mathrm{ZnWO}_{4}$ crystals [4].

The different contribution of the fast decay components in absorption and luminescence for oxygen deficient center can be due to different probabilities for corresponding electron transitions. Probabilities are different also for electron transitions responsible for the absorption and luminescence, if compared, for intrinsic luminescence centers and oxygen deficient centers. The $2.5 \mathrm{eV}$ band is dominant in the spectrum of the luminescence of yellow hue crystal excited by electron beam (Fig. 1, curve 3 ), whereas the $\approx 2.0 \mathrm{eV}$ band is dominant in the absorption spectrum (Fig. 3, curve 1).

One can estimate from the sum of luminescence and absorption peak energies $(2.0 \mathrm{eV}+2.0 \mathrm{eV}=4 \mathrm{eV})$, that upper energy level of the oxygen-deficient luminescence center is close to the bottom of the conduction band. Whereas the forbidden gap in CWO was estimated to be $\geq 5.2 \mathrm{eV}$ [5], the fundamental absorption edge tail due to oxygen deficient states or exciton states was observed at lower energies (Fig. 3, curves 4 and 5). From these estimations, one can draw out the approximate positions of levels of the ground state as well as excited state of the luminescence center. If the ground state of the luminescence center is assumed to be $0.2-0.6 \mathrm{eV}$ above the top of valence band, the rough calculations gives that the relaxed first excited state level is located about $2.2-2.6 \mathrm{eV}$ below the bottom of the conduction band. The second excited state level is located about $2.0 \mathrm{eV}$ above the first one.

\section{Conclusions}

The excited state absorption of the luminescence centers associated with oxygen deficient defects was observed. The upper energy level of this transition is located possibly below the bottom of the conduction band.

\section{Acknowledgments}

Work supported by Latvian SC (grant 96.0662).

\section{References}

[1] R. Deych, J. Dobbs, S. Marcovici, B. Tuval, in: Proc. Int. Conf. on Inorganic Scintillators and their Applications, Eds. P. Dorenbos, C.W.E. van Eijk, Delft University Press, Delft 1995, p. 36.

[2] L. Grigorjeva, R. Deych, D. Millers, S. Chernov, Radiat. Measur. 41, 267 (1998).

[3] D.R. Kinloch, W. Nowak, P. Raby, I. Toepke, IEEE Trans. Nucl. Sci. 41, 752 (1994).

[4] M.J.J. Lamers, G. Blasse, D.S. Robertson, Phys. Status Solidi A 63, 569 (1981).

[5] V. Nagirnij, E. Feldbach, L. Jönsson, M. Kirm, A. Lushchik, Ch. Lushchik, L.L. Nagornaya, V.D. Ryzhikov, F. Savikhin, G. Svensson, I.A. Tupitsina, $R a-$ diat. Measur. 41, 247 (1998). 